\title{
Experimental and Numerical Studies of Suppression of Forest Combustible Material Pyrolysis under Influence of Steam-Water Curtain
}

\author{
Dmitry Antonov ${ }^{1, *}$, Konstantin Osipov ${ }^{2}$, and Irek Khasanov ${ }^{3}$ \\ ${ }^{1}$ National Research Tomsk Polytechnic University, 634050 Tomsk, Russia \\ ${ }^{2}$ V.E. Zuev Institute of Atmospheric Optics SB RAS, 634055 Tomsk, Russia \\ ${ }^{3}$ All-Russian Research Institute for Fire Protection of Russian Emergency Ministry, 143903 Moscow \\ Region, Russia
}

\begin{abstract}
Forest fires inflict enormous damage to the environment and the economy of many countries around the world. This paper presents the results of experimental and numerical studies of the suppression of the pyrolysis reaction of forest combustible materials (birch leaves, spruce needles) under the influence of water vapor. The dependences of the characteristic times of suppression of the thermal decomposition and the complete decomposition of forest combustible material on the thickness of its layer were established. We used typical model of fire source with fixed height $0.04 \mathrm{~m}$ and varied diameter $0.02-0.06 \mathrm{~m}$. The processes of suppressing the pyrolysis reaction of forest combustible materials were recorded using a high-speed video camera (recording frequency $6 \cdot 10^{5} \mathrm{fps}$ ). Numerical studies were conducted using Ansys Fluent and Matlab. The obtained results are important for the development of technologies of extinguishing forest bottom fires.
\end{abstract}

\section{Introduction}

Wildfire fighting is an urgent problem for the entire world community [1-4]. Only in Russia the number of forest fires on average is about 30 thousand each year [5,6]. There are various ways to extinguish forest fires using water [1-4]. The use of water vapor is the most interesting from the standpoint of extinguishing forest bottom fires. The principle of operation is based on the fact that the water vapor creates a vapor shell above the combustion zone and separates it from the oxidant of the external medium, thereby stopping combustion. However, this method of fire extinguishing is poorly studied both experimentally and theoretically and requires detailed investigation.

The aim of this work is experimental and numerical studies of the suppression of the pyrolysis reaction of forest combustible materials under the influence of water vapor.

\footnotetext{
* Corresponding author: 1aadsdfn@gmail.com
} 


\section{Experimental setup and procedure}

Fig. 1 shows the scheme of experimental setup used in carrying out researches. As the main recording equipment we chose a high-speed digital CMOS video camera Phantom V411 1 with a recording frequency is of $6 \cdot 10^{6} \mathrm{fps}$ and maximum resolution of $1280 \times 1280$ pixels) and high-speed (the minimum polling rate is $0.1 \mathrm{~s}$ ) analog input module "National Instruments NI 9216" 2 for temperature sensors connection.

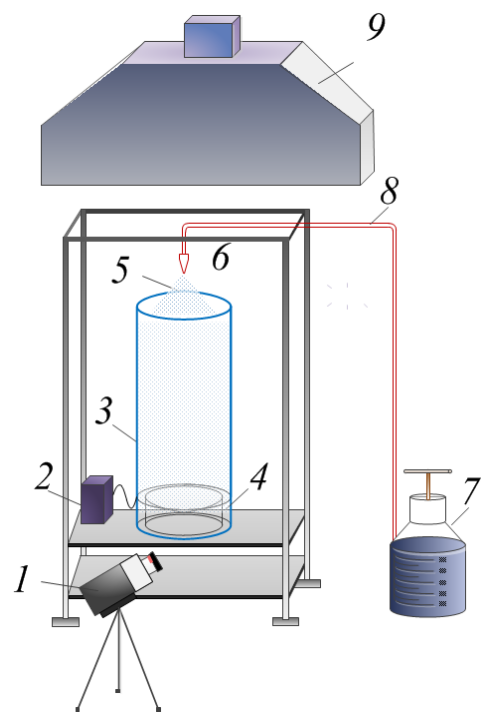

Fig. 1. A scheme of experimental setup: 1 - high-speed video camera; 2 - high-speed analog input module; 3 - cylinder with a forest material; 4 - metal tray; 5 - liquid aerosol; 6 - spraying nozzle; 7 bulb with quenching liquid; 8 - quenching liquid supply channel; 9 - exhaust system.

The experiments were carried out in two stages: the first one was used to determine the times of complete burning out of model sources of forest material burning $-\tau_{\mathrm{h}}$; the second one included recording the extinguishing times (suppression of flame burning and thermal decomposition) of model sources under water vapor extinguishing $-\tau_{\mathrm{d}}$.

Cylinder 3 with a sample of forest material was installed on a non-combustible metal tray 4 (Fig. 1). Next, we ignited the model fire seat uniformly over the entire area of the open surface of the forest material with the simultaneous use of three piezoelectric gas burners. The time recording began via the electronic stopwatch (the time step was $0.01 \mathrm{~s}$ ). The time of complete burnout $\left(\tau_{\mathrm{h}}\right)$ of the total volume of forest combustible material (the period of time from the onset of combustion to the moment of burning out) was recorded. At the second stage of the experiments, the extinguishing of the model fire seat was carried out.

During the experimental studies, a vapor-drop mixture (vapor-water cloud) with a predetermined temperature over the decomposing forest combustible material was created $\left(T_{\mathrm{m}}=300-450 \mathrm{~K}\right)$. We recorded the time during which the temperature of the heated forest combustible material became lower than the temperature of its thermal decomposition beginning. This value corresponded to the conditions for stopping the pyrolysis reaction of forest combustible material. The tested sample was a layer (filling the cylindrical channel 3) of a forest combustible material with a thickness from $0.02 \mathrm{~m}$ to $0.06 \mathrm{~m}$.

The systematic errors in determining the times $\tau_{\mathrm{h}}$ and $\tau_{\mathrm{d}}$ are $0.5 \mathrm{~s}$. The maximum random errors in determining the temperatures $T_{\mathrm{m}}$ during the thermal decomposition of forest combustible material did not exceed $30 \mathrm{~K}$. 


\section{Mathematical model}

Two software packages Ansys Fluent and Matlab were used when carrying out the numerical studies. These software packages differ primarily in the difference in the solution method. Matlab uses the finite difference method; Ansys Fluent uses the finite element method. In addition, our own code was created in Matlab with the refinement of all the terms of the system of differential equations of heat and mass transfer in partial derivatives.

The solved problem of determining the burning times and the extinguishing times of forest combustible material was two-dimensional. The thickness of the near-surface layer of forest combustible material was varied in the range $L_{\mathrm{f}}=35-80 \mathrm{~mm}$. The dimensions of the solution region were varied depending on the values of $L_{\mathrm{f}}$ in the ranges $H=0.1-0.5 \mathrm{~m}$ and $L=0.1-0.5 \mathrm{~m}$. The solution region of the problem under consideration for the system "gasvapor mixture - forest combustible material" is presented in Fig. 2.

Numerical analysis of the investigated physical and chemical processes is performed under the following assumptions, which do not impose significant restrictions on the generality of the problem formulation:

1.It was assumed that in a small neighborhood of a trace of a typical water mass, the concentration of the oxidizer is close to zero due to sufficiently intense vaporization.

2. It was believed that the vapor-gas mixture consists of water vapor $(90 \%)$ and combustion products $(10 \%)$.

3. We did not take into account possible oxidation reactions of pyrolysis products of forest combustible material when modeling the processes of heat transfer in the gas phase.

4. It was assumed that the thermophysical characteristics of forest material and water do not depend on temperature when setting the heat transfer problem.

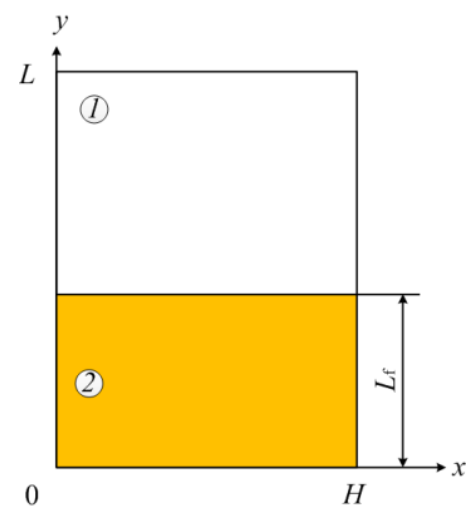

Fig. 2. Scheme of the region for solving the heat transfer problem at $0<t<\tau_{\mathrm{d}}\left(\tau_{\mathrm{h}}\right)$ for the system "vapor-gas mixture - forest combustible material"; 1 - vapor-gas mixture, 2 - forest combustible material.

The solution of the problem of heat and mass transfer using own code in Matlab is considered in detail in [7]. In this paper, we describe a system of partial differential equations for the considered system "vapor-gas mixture-water vapor - forest combustible material".

In the software complex Ansys Fluent, the solution of the heat and mass transfer problem consisted of several stages. The first stage was the creation of a grid model, which is an integral part of the process of mathematical modeling. The quality of the computational grid affects the accuracy, convergence and speed of the solution. In addition, the time to create a grid, often takes a significant proportion of the total execution time. In this paper, the time steps $\Delta t=0.1 \mathrm{~s}$ and the coordinate steps $\Delta x=\Delta y=0.05 \mathrm{~mm}$ were used to solve the heat and mass transfer task. Near the boundaries of the phase transition, the grid 
was thickened to improve the calculation accuracy (up to $\Delta x=\Delta y=\Delta r=0.001 \mathrm{~mm}$. The second stage was the description of the main used models (Energy Model, Specific Transport Model, Turbulence Model and e.t.).

To ensure high accuracy of calculations, the turbulence model corresponding to the formulated problem $k-\varepsilon$ was chosen [8]. It is a two-parameter model in which two transfer equations are solved to determine the turbulent velocity and the time of the vortex dissipation.

\section{Results and discussion}

Typical temperature fields at extinguishing forest combustible material by water vapor are presented in Fig. 3. The moments before the suppression of the forest combustible material thermal decomposition $(\tau=50 \mathrm{~s})$, in the process $(\tau=200 \mathrm{~s})$ and after completion $(\tau=500 \mathrm{~s})$ of thermal decomposition are shown.
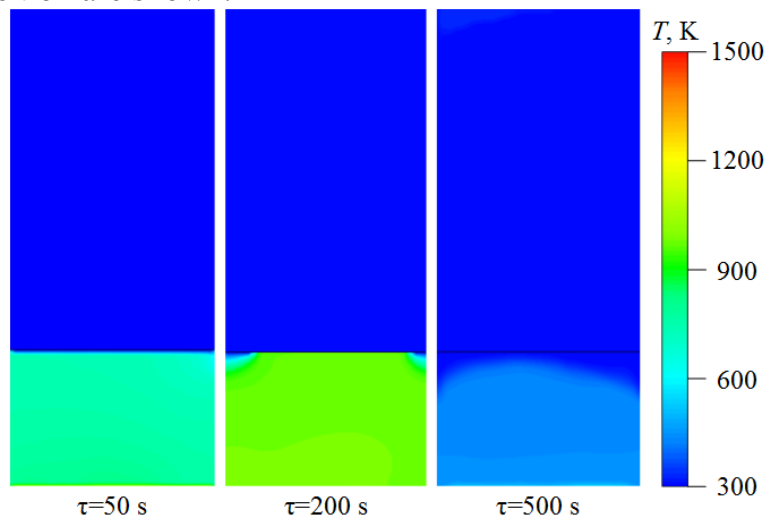

Fig. 3. Temperature fields during thermal decomposition of LCM at different instants of time.

Fig. 4 shows the dependence of the characteristic times of suppression of the thermal decomposition of forest combustible material $\left(\tau_{\mathrm{d}}\right)$ and the times of total forest combustible material thermal decomposition $\left(\tau_{\mathrm{h}}\right)$ as a function of the thickness of the forest combustible material layer. It should be noted the non-linear nature of the time variation as a function of $L_{\mathrm{f}}$. This is due to the nonlinear dependence of the thermal decomposition rate in the nearsurface layer of forest combustible material [3, 5, 9-12].

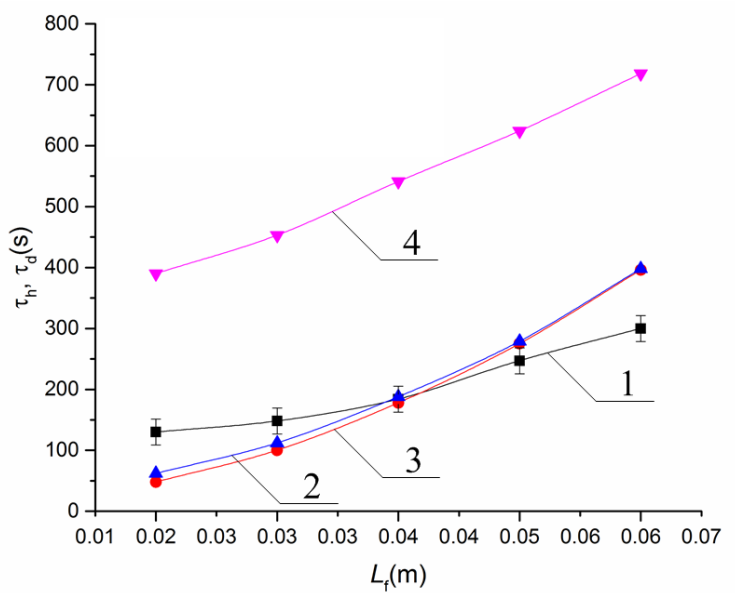

(a) 


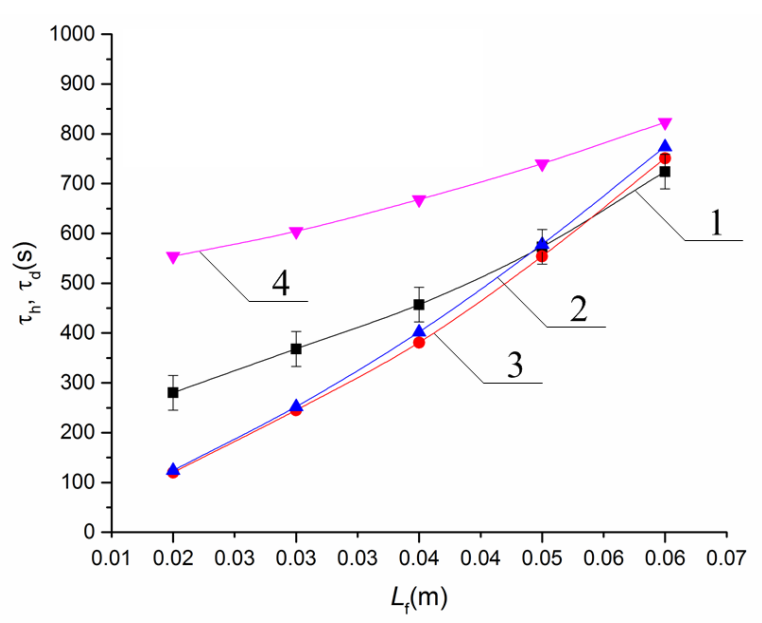

(b)

Fig. 4. Characteristic times of suppression of the thermal decomposition reaction of forest combustible material (birch leaves (a), spruce needles (b)): 1- experimental data, 2 - results of numerical modeling in Matlab, 3 - results of numerical modeling in Ansys Fluent, 4 - time of complete burning of LCM.

We have established a satisfactory agreement between the results of numerical calculations performed in software complexes (Matlab and Ansys Fluent) and experimental studies (Fig. 4). Deviations of the theoretical curves from the experimental ones can be explained by the fact that the constants $(\lambda, C$ and $\rho)$ were used as the values of thermophysical parameters when modeling. To approximate theoretical models to real applications, it is useful to consider the dependence of $\lambda, C$ and $\rho$ on moisture content and temperature.

In addition, it should be noted that the initial parameters of solving the heat transfer problem have a significant effect on the deviations of theoretical results. This is, first of all, the thermal decomposition kinetics (activation energy, pre-exponential factor, heat of combustion, initial temperature of water vapor).

We used the kinetics of thermal decomposition for deciduous and coniferous tree species $[1,3,5,9]$ in numerical simulation. From the analysis [1, 3, 5, 9] it can be concluded that forest materials of different regions differ in their thermophysical properties and kinetic parameters.

The effect of the change in the fragments of the samples from experiment to experiment is enhanced when carrying out experiments with large thicknesses of the reacting layer. Most likely, the position of the theoretical curve relative to the experimental curve is due precisely to this factor when the thickness of the sample of birch leaves is more than 0.05 $\mathrm{m}$. Such an effect is not observed with spruce needles, since the structure of the fragments is more uniform in comparison with the leaves. However, it was not possible to ensure the absolute identity of spruce needles from experiment to experiment.

\section{Conclusion}

As a result of the experimental and theoretical studies, we established the values of times of forest material (birch leaves, spruce needles) extinguishing and burning out depending on the thickness of the layer of forest combustible material. The obtained results can be used to predict the times of extinguishing forest bottom fires. 
The research was funded by the Russian Science Foundation (project 18-19-00056).

\section{References}

1. O. P. Korobeinichev, A. G. Shmakov, V. M. Shvartsberg, A. A. Chernov, S. A. Yakimov, K. P. Koutsenogii, V. I. Makarov, Fire Saf. J. 51, 102 (2012), DOI: 10.1016/j.firesaf.2012.04.003

2. C. C. Remy, M. Lavoie, M. P. Girardin, H. Asselin, A. A. Ali, J. Biogeogr. 1 (2017)

3. R. S. Volkov, A. O. Zhdanova, G. V. Kuznetsov, P. A. Strizhak, Tech. Phys. 60, 1443 (2015), DOI: 10.1134/S1063784215100291

4. D. Morvan, Fire Saf. J. 71, 34 (2015), DOI: 10.1016/j.firesaf.2014.11.012

5. A. O. Zhdanova, G. V. Kuznetsov, J. C. Legros, P. A. Strizhak, Thermal Sci. 21 (6A), 2559 (2017), DOI: 10.2298/TSCI151006121Z

6. O. Ulybina, Forest Policy and Economics, 38, 143 (2014), DOI: 10.1016/j.forpol.2013.06.019

7. A. O. Zhdanova, G. V. Kuznetsov, P. A. Strizhak, Applied Mechanics and Materials, 692, 267 (2014), DOI: 10.4028/www.scientific.net/AMM.692.267

8. A. D. Kliukvin, Science \& Education of Bauman MSTU, 8, 256 (2014), DOI: 10.7463/0814.0725648

9. A. I. Karpov, V. B. Novozhilov, A. A. Galat, V. K. Bulgakov, Fire safety science: proceeding of eight international symposium 27, 753 (2005), DOI: 10.3801/IAFSS.FSS.8-753

10. D. V. Barovik, Math. Modell. Anal. 15, 161 (2010), DOI: 10.3846/13926292.2010.15.161-174

11. E. Pastor, L. Zarate, E. Planas, J. Arnaldos, Prog. Energy Combust. Sci. 29, 139 (2003), DOI: 10.1016/S0360-1285(03)00017-0

12. J. Qie, L. Yang, Y. Wang, J. Dai, X. Zhou, J. Fire Sci. 29, 243 (2011), DOI: 10.1177/0734904110392961 